\title{
Perfil epidemiológico da mortalidade em um hospital de urgência em Sergipe Epidemiological profile of mortality in a hospital of urgency in Sergipe
}

\author{
Áquila Talita Lima Santana Alves ${ }^{1}$, André Faro ${ }^{2}$ \\ 1. Estudante de Medicina pela Universidade Federal de Sergipe (UFS) e bolsista PET-Saúde/RUE, São Cristóvão, SE, Brasil. ORCID: http://orcid.org/0000- \\ 0002-1352-6795. 2. Docente do Departamento de Psicologia da Universidade Federal de Sergipe (UFS) e tutor do PET-Saúde/RUE, São Cristóvão, SE, Brasil.
} ORCID: http://orcid.org/0000-0002-7348-6297

\begin{abstract}
Resumo
Objetivos: caracterizar o perfil da mortalidade em um Hospital de Urgências de Sergipe nos anos 2013 e 2014 e analisar a relação entre a Causa Básica e a Causa Imediata do óbito. Métodos: estudo descritivo e exploratório, realizado a partir do levantamento de informações do banco de dados da Organização de Procura de Órgãos e Tecidos da Instituição. Obtiveram-se as estatísticas descritivas para a caracterização do perfil amostral por idade, sexo, ano do óbito, setor do hospital e causas do óbito. Para a avaliação da relação entre a Causa Básica e a Causa Imediata de óbito, analisaram-se valores absolutos e percentuais quanto aos tipos de entrada (Causa Básica) e ao desfecho (Causa Imediata). Resultados: entre 5.455 óbitos, 57,6\% foram do sexo masculino e a média de idade foi de 55,7 anos $( \pm 23,5$ ). As Doenças Infecciosas e as Causas Mal Definidas representaram quase metade dos óbitos na Causa Imediata 1 $(48,3 \%)$. A relação entre Causa Imediata e Básica mostrou que as Doenças Infecciosas são a principal Causa Imediata de óbitos em oito entre onze categorias da Causa Básica. Conclusão: as Doenças Infecciosas e as Causas Mal Definidas exibem notável impacto na mortalidade hospitalar. Além disso, os dados obtidos neste estudo sugerem a viabilidade do uso do registro da Organização de Procura de Órgãos e Tecidos como parâmetro de avaliação da mortalidade hospitalar.
\end{abstract}

Palavras-chave: Mortalidade Hospitalar. Causas de Morte. Infecção Hospitalar

\begin{abstract}
Objectives: characterizing the mortality profile in an Urgency Hospital of Sergipe in the years 2013 and 2014 and to analyzing the relationship between the Underlying Causes and the Immediate Cause of death. Methods: a descriptive and exploratory study targeted at collecting information from the institutional database of the Organ and Tissue Procurement Organization. We obtained descriptive statistics to characterize the sample profile for age, sex, year of death, hospital sector and death causes. For the assessment of the relationship between the Underlying Causes and the Immediate Cause of death, we analyzed the absolute and percentage values by the type of input (Underlying Cause) and outcome (Cause Immediate). Results: From 5455 deaths, $57.6 \%$ were male and mean age was 55.7 years $( \pm 23.5)$. Infectious Diseases and ill-defined causes accounted for almost half of the deaths in the Immediate Cause $1(48.3 \%)$. The relationship between Immediate and Basic Causes showed that infectious diseases are the main Immediate causes of death in eight of eleven categories of the Basic Cause. Conclusion: the Infectious Diseases and Ill-defined Causes exhibited remarkable impact on hospital mortality. In addition, the study data suggests the feasibility of the Organ and Tissue Procurement Organization`s information as an evaluation parameter for hospital mortality.
\end{abstract}

Keywords: Hospital Mortality. Causes of Death. Cross Infection.

\section{INTRODUÇÃO}

Identificar quantas pessoas morrem a cada ano e por que morrem é um dos meios mais importantes para avaliar a eficácia do sistema de saúde de um país ${ }^{1}$. O estudo da mortalidade é um mecanismo para se conhecer uma sociedade, pois por meio da avaliação do nível e do padrão de mortalidade é possível analisar as condições sociais e econômicas e quais as suas demandas políticas e sociais ${ }^{2}$. Ademais, conhecer as causas de morte ajuda as autoridades de saúde a determinar seu foco para ações de saúde pública ${ }^{1,2}$.

No Brasil, os dados de mortalidade hospitalar podem ser consultados através do Sistema de Informação sobre Mortalidade (SIM), por meio da Declaração de Óbito (DO), que é o seu documento padrão. Outro sistema de informação que pode ser consultado para esse fim é o Sistema de Informações Hospitalares Descentralizado do Sistema Único de Saúde (SIHD/ SUS), que armazena dados sobre as internações hospitalares do SUS, cujo documento padrão é a Autorização de Internação
Hospitalar (AIH). O SIHD possibilita o conhecimento do perfil de mortalidade hospitalar por meio do número de internações que tiveram alta por óbito.

Algo que há em comum entre os dois sistemas (SIM e SIHD) são os problemas no registro de informação, gerando um considerável volume de perdas e comprometendo a qualidade do registro, o que dificulta a geração de dados confiáveis a respeito da mortalidade ${ }^{3}$. Principalmente por esse motivo, podem ser pensadas diferentes alternativas para obter dados mais fidedignos, sendo que uma delas é a utilização de registros internos às instituições, pois trata-se de controles logísticos próprios ou assistenciais, nem sempre disponibilizados no DATASUS ou em sítios similares ${ }^{4,5}$.

Tendo em vista as limitações apresentadas pelo SIM e pelo SIHD, principalmente no que diz respeito ao preenchimento inadequado da DO e considerando a possibilidade de se usarem

Correspondência: Áquila Talita Lima Santana Alves. Rua Jasiel de Brito Cortes, Residencial Vila Velha 20, Bloco 3 apto 104 - Bairro Jabotiana. CEP: 49095780 Aracaju, SE, Brasil. E-mail: aquilalima2@gmail.com

Conflito de interesse: Não há conflito de interesse por parte de qualquer um dos autores.

Recebido em:3 Abr 2016; Revisado em: 25 Abr 2016 Aceito em: 10 Maio 2016 
fontes paralelas e confiáveis, julgou-se viável a coleta de dados no banco de dados da Organização de Procura de Órgãos e Tecidos (OPO). À semelhança da DO, essa fonte disponibiliza dados sociodemográficos e clínicos com a vantagem de utilizar mais de um instrumento (DO, AlH e prontuário) para o seu preenchimento. Isso, por sua vez, permite o cruzamento de informações, que suprem lacunas encontradas nas fontes tradicionais e trazendo um diferente panorama de avaliação da mortalidade.

Ao passo da necessidade de investigações a respeito da mortalidade e tendo em vista que o Hospital de Urgências de Sergipe (HUSE) é o principal hospital de atenção à média e alta complexidade do Estado, entende-se como pertinente retratar e caracterizar a mortalidade dentro desta Instituição. Além disso, considerando o diferencial da coleta dos dados com o registro da OPO, espera-se que com outra fonte sejam produzidos dados diferenciados que minimizem perdas comuns aos sistemas de informação de mortalidade.

Frente ao exposto, este estudo objetivou: 1. Levantar o perfil epidemiológico da mortalidade do HUSE nos anos 2013 e 2014 a partir da avaliação dos dados da OPO desse hospital; 2. Caracterizar a mortalidade segundo variáveis sociodemográficas (sexo e idade) e clínicas (causas de morte) e 3. Analisar a relação entre a Causa Básica e a Causa Imediata do óbito.

\section{MÉTODO}

\section{Local de estudo}

Localizado na capital do Estado de Sergipe, o Hospital de Urgência de Sergipe Governador João Alves Filho (HUSE) é o maior hospital público e principal porta de entrada do SUS para os casos de média e alta complexidade de Sergipe e cidades de estado vizinhos como Alagoas e Bahia, atendendo a uma média mensal de 15 mil usuários. Além disso, é um hospital de ensino, centro de referência de atendimento a vítimas de trauma e demais agravos à saúde. Atualmente, o estabelecimento compreende a maior urgência e emergência do Estado e quatro espectros: cirúrgico, clínico, complementar e pediatria clínica. Sua capacidade física instalada é de 567 leitos.

\section{Desenho de estudo e coleta de dados}

Estudo descritivo e exploratório. As informações foram selecionadas do banco de dados da OPO, que disponibiliza as seguintes variáveis: data e hora do óbito, número de registro hospitalar, setor do hospital que ocorreu o óbito, nome do paciente, idade, causas de morte, sexo, realização de entrevista para captação de órgãos e tecidos, motivo da não doação. Entre essas variáveis, não foram utilizadas na presente pesquisa apenas as informações referentes à doação de órgãos e tecidos.

\section{Procedimentos}

Os responsáveis por alimentar o banco de dados da OPO extraem as causas de morte através da busca ativa na área do hospital que ocorreu o óbito, e são listadas, em geral, 5 causas para cada paciente, padronizadas segundo o Manual de preenchimento da $\mathrm{DO}^{6}$. Causa 1 como causa imediata de morte (causa que provocou a morte), Causas 2 e 3 como causas consequenciais (estados mórbidos que produziram a causa imediata), Causa 4 como causa básica, e Causa 5 como causa contribuinte (outras patologias que contribuíram para a morte).

Ao finalizar a coleta levantou-se um total de 597 causas de morte. Foram encontrados desde registros de resultados laboratoriais (por exemplo, leucocitose), a parada cardíaca ou causa indeterminada. Por isso, inicialmente, buscou-se a redução mediante categorização de causas em comum, utilizando-se como referência o CID-107. Procederam-se duas rodadas de agrupamentos e ao final obtiveram-se 18 categorias, a saber:

1. Doenças infecciosas (por exemplo, infecções de partes moles, incluindo sepse e choque séptico);

2. Doenças parasitárias;

3. Choques (excluindo choque séptico);

4. Causas externas;

5. Neoplasias (incluindo metástases);

6. Causas cirúrgicas;

7. Causas mal definidas (causa indeterminada, SVO, achados laboratoriais);

8. Doenças do aparelho respiratório;

9. Doenças do aparelho circulatório;

10. Doenças do sistema nervoso;

11. Doenças do aparelho geniturinário;

12. Doenças endócrinas, nutricionais e síndromes genéticas;

13. Doenças do aparelho digestivo;

14. Doenças da pele;

15. Doenças do sangue e órgãos hematopoiéticos;

16. Causas relacionadas a gravidez, parto e puerpério;

17. Doenças do sistema osteomuscular; e

18. Doenças psiquiátricas.

$\mathrm{Na}$ categorização final foram alcançados cinco grandes grupos e as causas que apresentaram frequência menor que $1 \%$ em cada grupo foram nomeadas como Outras. Para a apresentação das variáveis, a fim de tornar claro o registro da categoria por Causa na descrição dos resultados, cada sigla de motivo será acompanhada por um número que se refere à classificação da Causa registrada na OPO. Logo, para as categorias da Causa 1, todas as siglas estão seguidas do número 1 (por ex., INF1). A mesma categoria, quando registrada na Causa 3, está abreviada com o numeral correspondente a essa Causa (por ex. INF3).

a. Causa 1 ou imediata - com 12 categorias: doenças infecciosas (INF1), choque (CHQ1), neoplasias (NEO1), doenças do aparelho respiratório (DAR1), doenças do aparelho circulatório (DAC1), doenças do sistema nervoso (DSN1), doenças do aparelho geniturinário (DAG1), doenças endócrinas, nutricionais e síndromes genéticas (DEN1), doenças do aparelho digestivo (DAD1), causas mal definidas (CMD1), doenças do sangue e órgãos hematopoiéticos (DSH1) e Outras (OUT1). 
b. Causa 2 ou consequencial - com 10 categorias: doenças infecciosas (INF2), doenças do aparelho respiratório (DAR2), doenças do aparelho circulatório (DAC2), doenças do sistema nervoso (DSN2), doenças do aparelho geniturinário (DAG2), doenças endócrinas, nutricionais e síndromes genéticas (DEN2), doenças do aparelho digestivo (DAD2), causas mal definidas (CMD2), doenças do sangue e órgãos hematopoiéticos (DSH2) e Outras (OUT2).

c. Causa 3, também consequencial - com 11 categorias: doenças infecciosas (INF3), causas externas (CEX3), neoplasias (NEO3), doenças do aparelho respiratório (DAR3), doenças do aparelho circulatório (DAC3), doenças do sistema nervoso (DSN3), doenças do aparelho geniturinário (DAG3), doenças endócrinas, nutricionais e síndromes genéticas (DEN3), doenças do aparelho digestivo (DAD3), doenças do sangue e órgãos hematopoiéticos (DSH3) e Outras (OUT3).

d. Causa 4 ou básica - com 11 categorias: doenças infecciosas (INF4), causas externas (CEX4), neoplasias (NEO4) e, doenças do aparelho respiratório (DAR4), doenças do aparelho circulatório (DAC4), doenças do sistema nervoso (DSN4), doenças do aparelho geniturinário (DAG4), doenças endócrinas, nutricionais e síndromes genéticas (DEN4), doenças do aparelho digestivo (DAD4), doenças do sangue e órgãos hematopoiéticos (DSH4) e Outras (OUT4).

e. Causa 5 ou contribuinte - com 5 categorias: neoplasias (NEO5), doenças do aparelho circulatório (DAC5), doenças do aparelho geniturinário (DAG5), doenças endócrinas, nutricionais e síndromes genéticas (DEN5); e Outras (OUT5).

\section{Análise dos dados}

A partir do levantamento das informações foi construído um banco de dados no programa SPSS (v.19), tendo sido obtidas a estatística descritiva para caracterização do perfil da amostra por idade, sexo, ano do óbito, setor do hospital e causas do óbito. Para a avaliação das relações entre a Causa Básica (Causa 4; Linhas) e Causa Imediata de óbito (Causa 1; Colunas), construiu-se uma Tabela em que constam os valores absolutos e percentuais entre os tipos de entrada (Causa Básica) e desfecho (Causa Imediata). Assim, quanto maior o índice percentual, maior a quantidade de casos em que o tipo de entrada (Causa Básica) evoluiu para essa Causa Imediata de óbito.

\section{Considerações éticas}

O estudo foi submetido ao Comitê de Ética em Pesquisa em Seres Humanos da Universidade Federal de Sergipe (UFS) e aprovado de acordo com a CAAE 32954114.1.0000.5546. Além disso, foi autorizado pelo Núcleo de Educação Permanente da Instituição e pela gerente da OPO. Todos os procedimentos éticos propostos e aprovados foram rigorosamente seguidos pela equipe de pesquisa.

\section{RESULTADOS}

Entre janeiro de 2013 e dezembro de 2014 foram registrados 5455 óbitos, sendo 51\% ( $n=2783)$ em 2013 e 49\% $(n=2672)$ em 2014. Os meses de maior registro foram março e dezembro de 2013 , ambos com 4,7\% ( $n=257)$ e o de menor registro foi fevereiro de 2014 com 3,3\% ( $n=185)$ do total de óbitos. A média mensal de óbitos, ao longo dos dois anos, foi 227,2 (DP+21,7), tendo uma média de 231,9 (DP+20,0) para 2013 e 222,6 $(D P+23,2)$ para 2014.

Vale salientar que houve diferença entre a quantidade de dados perdidos (Perdas) por variável estudada. Sendo assim, a descrição dos dados será apresentada discriminando a quantidade de Perdas em cada variável e, posteriormente, indicar-se-ão os valores ajustados (sem Perdas) nas Tabelas de análise.

Dos 5455 óbitos, $57,6 \%$ ( $n=3146$ ) foram do sexo masculino e $42,3 \%$ ( $n=2309$ ) do feminino, não havendo perda nessa variável. Em relação à idade, a média foi de 55,7 anos (DP \pm 23,5; range $=0-108$ ). O agrupamento por faixas etárias mostrou que a maior parte $(48,7 \% ; n=2657)$ pertencia ao grupo acima de 60 anos. Ao se comparar a faixa etária por sexo, verificouse que a mortalidade para o sexo masculino nas faixas etárias entre 13 e 18 anos e entre 19 e 35 anos foi cerca de duas vezes maior do que o encontrado no sexo feminino (Tabela 1 ).

Tabela 1. Distribuição dos óbitos ( $n=5455)$ de acordo com sexo e faixa etária. Hospital de Urgências de Sergipe (HUSE), 20132014

\begin{tabular}{lrrr}
\hline \multirow{2}{*}{ Faixa etária } & Geral & Masculino & Feminino \\
\cline { 2 - 4 } & $\mathbf{n}(\%)$ & $\mathbf{n}(\%)$ & $\mathbf{n}(\%)$ \\
\hline 0 a 12 anos & $315(5,8)$ & $166(5,3)$ & $149(6,5)$ \\
13 a 18 anos & $126(2,3)$ & $94(3,0)$ & $32(1,4)$ \\
19 a 35 anos & $675(12,4)$ & $494(15,7)$ & $181(7,8)$ \\
36 a 60 anos & $1682(30,8)$ & $994(31,6)$ & $688(29,8)$ \\
Acima de 60 & $2657(48,7)$ & $1398(44,4)$ & $1259(54,5)$ \\
Total & $5455(100,0)$ & $3146(100,0)$ & $2309(100,0)$ \\
\hline
\end{tabular}

O setor com o número mais elevado de óbitos foi o ProntoSocorro/Adulto com $58,6 \%(n=3198)$ (Tabela 2). O maior valor de Perdas entre as Causas de óbito foi de $90 \%(n=4907)$ para as Causas contribuintes (Causa 5 ) e o menor valor foi nas Causas Básicas (Causa 4) $(10,9 \%, n=592)$.

Tabela 2. Distribuição dos óbitos $(n=5455)$ de acordo com o setor do Hospital. HUSE, 2013-2014

\begin{tabular}{lr}
\hline \multicolumn{1}{c}{ Área } & $\mathrm{n}(\%)$ \\
\hline Pronto-Socorro/Adulto & $3198(58,6)$ \\
Internamento/Adulto & $959(17,6)$ \\
UTI'1/Adulto & $767(14,1)$ \\
UTI'1/Pediátrica & $87(1,6)$ \\
Centro Cirúrgico & $245(4,5)$ \\
Pronto-Socorro/Pediátrico & $157(2,9)$ \\
\hline
\end{tabular}




\begin{tabular}{lr}
\hline \multicolumn{1}{c}{ Área } & $\mathbf{n}(\%)$ \\
\hline Internamento/Pediátrico & $20(0,4)$ \\
UTQ $^{2}$ & $15(0,3)$ \\
Via SAMU & $7(0,1)$ \\
Total & $5455(100)$ \\
\hline
\end{tabular}

1 UTI-Unidade de Terapia Intensiva.

2 UTQ - Unidade de Tratamento de Queimados.

3 Via SAMU-paciente trazido ao hospital via Serviço de Atendimento Móvel de Urgência (SAMU) evoluiu a óbito antes de ser alocado em algum setor do hospital.

A causa com maior quantidade de óbitos validados ( $n=4863$ ) foi a Básica (Causa 4), em que a principal categoria responsável pelos óbitos foi a NEO4 (18,6\%; n = 903). Observou-se na Causa Imediata (Causa 1) o predomínio da INF1 e CMD1, representando $30,7 \%$ ( $n=1476)$ e $17,6 \%(n=847)$, respectivamente, que, quando somadas, compuseram quase metade do total de óbitos (48,3\%; $n=2323$ ) (Tabela 3).

A Tabela 4 fornece os dados da relação entre o motivo de entrada no serviço hospitalar e o desfecho - ou seja, a relação entre as Causas Básica (Causa 4) e Imediata (Causa 1) -, com os valores percentuais calculados para a Causa Básica (Causa 4). A partir da relação estabelecida, detectaram-se 4220 casos de óbito em que ambas as variáveis foram preenchidas, assim, esse é o número total de associações contidas na Tabela. A categoria INF1 é a principal Causa Imediata (Causa 1) em oito das onze categorias da Causa Básica (Causa 4). Em duas delas, as INF1 representaram mais de $50 \%$ do número de óbitos: INF4 $(62,4 \% ; n=333)$ e DAR4 $(60,7 \% ; n=247)$. A Causa Imediata (Causa 1) CMD1 teve índice maior que $10 \%$ em oito das onze Causas Básicas (Causa 4) de óbito, sendo que para a Causa Básica (Causa 4) NEO4, foram 18,6\% (n=137) dos casos (Tabela 4).

\section{DISCUSSÃO}

Os resultados mostraram que o total de óbitos registrados em 2013 foi de 2783 casos e em 2014 foi de 2672 casos. Ao se comparar com os dados fornecidos pelo DATASUS, chamou a atenção a discrepância de valores encontrados para esses mesmos anos, pois em 2013 foram registrados apenas 1466 e em 2014 registraram-se 1083 óbitos. Isso reforça o que já foi apontado por alguns autores em relação ao problema no registro dos sistemas de informação oficiais, gerando um considerável volume de perdas ${ }^{3}$.

É importante destacar que, nesta investigação, foi encontrado

Tabela 3. Distribuição absoluta e relativa das Causas de óbito segundo categorias. HUSE, 2013-2014.

\begin{tabular}{|c|c|c|c|c|c|}
\hline $\mathrm{n}(\%)^{2,3}$ & Causa Imediata (1) & Causa Consequencial (2) & Causa Consequencial (3) & Causa Básica (4) & Causa Contribuinte (5) \\
\hline INF & $1476(30,7)$ & $442(25,5)$ & $273(14,2)$ & $550(11,3)$ & - \\
\hline $\mathrm{CHQ}$ & $434(9,0)$ & - & - & - & - \\
\hline CEX & - & - & $410(21,4)$ & $813(16,7)$ & - \\
\hline NEO & $145(3,0)$ & - & $128(6,7)$ & $903(18,6)$ & $54(9,9)$ \\
\hline DAR & $700(14,6$ & $397(22,9)$ & $212(11,1)$ & $412(7,6)$ & - \\
\hline DAC & $345(7,2)$ & $171(9,9)$ & $251(13,1)$ & $756(15,5)$ & $113(20,6)$ \\
\hline DSN & $291(6,1)$ & $135(7,8)$ & $124(6,5)$ & $320(6,6)$ & - \\
\hline DAG & $187(3,9)$ & $117(6,7)$ & $108(5,6)$ & $192(3,9)$ & $74(13,5)$ \\
\hline DEN & $95(2,0)$ & $62(3,6)$ & $63(3,3)$ & $320(6,6)$ & $158(28,8)$ \\
\hline DAD & $122(2,5)$ & $106(1,9)$ & $150(7,8)$ & $336(6,9)$ & $158(28,8)$ \\
\hline CMD & $847(17,6)$ & $54(3,1)$ & - & - & - \\
\hline DSH & $106(2,2)$ & $90(5,2)$ & $86(4,5)$ & $86(1,8)$ & - \\
\hline OUT & $61(1,3)$ & $161(9,3)$ & $112(5,8)$ & $175(3,6)$ & - \\
\hline Total sem perdas & 4809 (100) & $1735(100)$ & $1917(100)$ & $4863(100)$ & $548(100)$ \\
\hline Perdas na variável ${ }^{1}$ & $646(11,8)$ & $3720(68,2)$ & $3538(64,9)$ & $592(10,9)$ & 4907 (90) \\
\hline
\end{tabular}

10 percentual das perdas foi calculado em relação ao total de óbitos $(n=5455)$.

2 INF = Doenças Infecciosas; $\mathrm{CHQ}=$ Choque; CEX = Causas Externas; NEO = Neoplasias; DAR = Doenças do Aparelho Respiratório; DAC = Doenças do Aparelho Circulatório; DSN = Doenças do Sistema Nervoso; DAG = Doenças do Aparelho Geniturinário; DAD = Doenças do Aparelho Digestivo; CMD = Causas Mal Definidas; DEN = Doenças Endócrinas, Nutricionais, Metabólicas e Síndromes Genéticas; DSH = Doenças do Sangue, Órgãos Hematopoéticos, e Transtornos Imunitários; OUT = Outras.

30 sinal (-) denota que, para determinada Causa, essa categoria se enquadrou no grupo Outras. 
Tabela 4. Distribuição absoluta e relativa da relação entre a Causa Básica (4) e a Causa Imediata (1). HUSE, 2013-2014.

\begin{tabular}{|c|c|c|c|c|c|c|c|c|c|c|c|c|c|}
\hline \multirow{2}{*}{$\frac{\mathbf{N}(\%)^{\mathbf{1 , 2}}}{\text { Causa } 4}$} & \multicolumn{13}{|c|}{ Causa 1} \\
\hline & INF1 & CHQ1 & NEO1 & DAR1 & DAC1 & DSN1 & DAG1 & DEN1 & DAD1 & CMD1 & DSH1 & OUT1 & $\mathrm{TOT}^{2}$ \\
\hline INF4 & $333(62,4)$ & $10(1,8)$ & $0(0,0)$ & $64(12,0)$ & $13(2,4)$ & $5(0,9)$ & $21(3,9)$ & $8(1,5)$ & $6(1,1)$ & $56(10,5)$ & $11(2,0)$ & $6(1,1)$ & $533(100)$ \\
\hline CEX4 & $95(25,1)$ & $86(22,8)$ & $2(0,5)$ & $46(12,2)$ & $15(4,0)$ & $24(6,3)$ & $5(1,3)$ & $3(0,8)$ & $19(5,0)$ & $40(10,6)$ & $9(2,4)$ & $34(9,0)$ & $378(100)$ \\
\hline NEO4 & $130(17,6)$ & $34(4,6)$ & $96(13,0)$ & $170(23,1)$ & $24(3,3)$ & $14(1,9)$ & $53(7,2)$ & $35(4,7)$ & $31(4,2)$ & $\begin{array}{l}137 \\
(18,6)\end{array}$ & $13(1,8)$ & $0(0,0)$ & 737 (100) \\
\hline DAR4 & $247(60,7)$ & $8(2,0)$ & $0(0,0)$ & $80(19,7)$ & $6(1,5)$ & $2(0,5)$ & $10(2,5)$ & $7(1,7)$ & $2(0,5)$ & $42(10,3)$ & $3(0,7)$ & $0(0,0)$ & 407 (100) \\
\hline DAC4 & $150(20,0)$ & $75(10,0)$ & $3(0,4)$ & $124(16,5)$ & $205(27,3)$ & $98(13,1)$ & $20(2,7)$ & $6(0,8)$ & $9(1,2)$ & $52(6,9)$ & $7(0,9)$ & $1(0,1)$ & 750 (100) \\
\hline DSN4 & $96(30,2)$ & $10(3,1)$ & $0(0,0)$ & $70(22,0)$ & $7(2,2)$ & $79(24,8)$ & $5(1,6)$ & $3(0,9)$ & $1(0,3)$ & $43(13,5)$ & $3(0,9)$ & $1(0,3)$ & $318(100)$ \\
\hline DAG4 & $70(36,6)$ & $11(5,8)$ & $0(0,0)$ & $35(18,3)$ & $14(7,3)$ & $6(3,1)$ & $16(8,4)$ & $15(7,9)$ & $3(1,6)$ & $16(8,4)$ & $4(2,1)$ & $1(0,5)$ & $191(100)$ \\
\hline DEN4 & $114(35,7)$ & $21(6,6)$ & $4(1,3)$ & $31(9,7)$ & $35(11,0)$ & $33(10,3)$ & $20(6,3)$ & $6(1,9)$ & $4(1,3)$ & $42(13,2)$ & $5(1,6)$ & $4(1,3)$ & $319(100)$ \\
\hline DAD4 & $114(34,9)$ & $41(12,5)$ & $2(0,6)$ & $31(9,5)$ & $3(0,9)$ & $9(2,8)$ & $17(5,2)$ & $3(0,9)$ & $\begin{array}{l}34 \\
(10,4)\end{array}$ & $41(12,5)$ & $31(9,5)$ & $1(0,3)$ & $327(100)$ \\
\hline DSH4 & $5(5,9)$ & $43(50,6)$ & $0(0,0)$ & $6(7,1)$ & $0(0,0)$ & $0(0,0)$ & $1(1,2)$ & $3(3,5)$ & $0(0,0)$ & $10(11,8)$ & $\begin{array}{l}17 \\
(20,0)\end{array}$ & $0(0,0)$ & \\
\hline OUT4 & $58(33,1)$ & $48(27,4)$ & $0(0,0)$ & $26(14,9)$ & $8(4,6)$ & $5(2,9)$ & $5(2,9)$ & $3(1,7)$ & $6(3,4)$ & $12(6,9)$ & $3(1,7)$ & $1(0,6)$ & \\
\hline
\end{tabular}

${ }^{1} \mathrm{INF}=$ Doenças Infecciosas; $\mathrm{CHQ}=$ Choque; CEX = Causas Externas; NEO = Neoplasias; DAR = Doenças do Aparelho Respiratório; DAC = Doenças do Aparelho Circulatório; DSN = Doenças do Sistema Nervoso; DAG = Doenças do Aparelho Geniturinário; DAD = Doenças do Aparelho Digestivo; CMD = Causas Mal Definidas; DEN = Doenças Endócrinas, Nutricionais, Metabólicas e Síndromes Genéticas; DSH = Doenças do Sangue, Órgãos Hematopoiéticos, e Transtornos Imunitários; OUT = Outras; TOT $=$ Total

${ }^{2} \mathrm{O}$ “N” difere da amostra geral por representar apenas os óbitos em que as variáveis Causa 1 e Causa 4 foram preenchidas.

um grande volume de Perdas nos registros da OPO, sendo que, para algumas Causas, ultrapassaram-se os $50 \%$ dos casos (Causas 2,3 e 5) e em relação à Causa Contribuinte observou-se $90 \%$ de Perdas. Outros autores apontaram resultados semelhantes ${ }^{8,9} \mathrm{e}$ isso mostra que os esforços em melhorar a cobertura do registro de óbito no país, saltando de $80 \%$ em 1980-1991 para mais de $95 \%$ entre $2000-2010^{10}$ não foi acompanhado, necessariamente, de melhora na qualidade desse registro.

O alto número de perdas não é exclusividade brasileira ${ }^{4,5}$, persistindo a necessidade de se enfatizar o correto preenchimento de dados em saúde. No que concerne às Causas Contribuintes ${ }^{5}$, é importante destacar que se verificou o maior valor de Perdas entre as causas de óbito (90\%). Vale ressaltar que o preenchimento adequado de todos os campos referentes às causas de morte permitem conhecer a trajetória clínica e o encadeamento patológico que resultou no óbito. Inclusive, isso favorece a avaliação do impacto que as estratégias preventivas na atenção primária podem causar no perfil da mortalidade (em especial a Causa 5$)^{10}$.

Quanto às características sociodemográficas, o maior percentual de óbitos foi do sexo masculino $(57,6 \%)$, o que acompanhou outros trabalhos na temática, tanto nacionais: $54,5 \%$, em hospitais do SUS do Rio Grande do Sul ${ }^{11}$ e 55,9\% na UTI de um Hospital Regional do Distrito Federal ${ }^{12}$, como internacionais: $55,7 \%$ de mortalidade no sexo masculino em 192 hospitais de urgência em 59 países de diversos continentes, predominantemente entre os jovens (abaixo dos 35 anos) ${ }^{13}$. Ademais, vale salientar que o agrupamento por faixas etárias mostrou que $48,7 \%$ dos óbitos ( $n=2657$ ) ocorreram no estrato acima de 60 anos, mostrando que no HUSE a mortalidade de idosos acumula cerca de $50 \%$ do total de óbitos.

Como era esperado ${ }^{11}$, o setor do hospital com o número mais elevado de óbitos foi o Pronto-Socorro/Adulto. Um trabalho avaliando hospitais da rede SUS em um Estado brasileiro apontou que a maior taxa de mortalidade se deu quando a internação foi no setor de Emergência ${ }^{11}$. Ao se avaliarem as Causas Básicas de morte (Causa 4), observou-se que a principal categoria foi Neoplasias (18,6\%; $n=903)$. As neoplasias tornaram-se um importante problema de saúde pública em todo o mundo ${ }^{14,15}$, e é a segunda principal causa de óbito tanto em países desenvolvidos como em desenvolvimento ${ }^{16}$.

Não obstante aos desafios decorrentes do seu papel como referência de alta complexidade para trauma, os resultados apresentados neste estudo evidenciam a relevância do HUSE na área da assistência oncológica, impactando não apenas o fluxo de atendimentos, mas na assistência hospitalar e gestão exclusiva de recursos para essa finalidade ${ }^{17}$.

A segunda principal categoria das Causas Básicas de óbito foi Causas Externas $(16,7 \% ; n=813)$, refletindo, em certa medida, o cenário nacional, pois as causas externas ocupam lugar de destaque no perfil da morbimortalidade do país ${ }^{16,18}$. Sobre isso, já está documentada a evolução das hospitalizações por essa causa em hospitais públicos brasileiros ao longo de 10 anos (2002 a 2011), havendo progressivo aumento da demanda de internação hospitalar ${ }^{19}$. Em relação às Causas Consequenciais (causas 2 e 3), observou-se maior representatividade das Doenças Infecciosas (25,5\%) e Causas Externas (21,4\%). 
Embora o perfil de mortalidade hospitalar até aqui apresentado esteja relacionado com as características assistenciais do HUSE na região, é importante que se foque o alto índice de óbitos associados à infecção. Sobre isso, viu-se neste estudo que $30,7 \%$ ( $n=1476$ ) das Causas Imediatas de óbito foram explicadas por infecção, com valores superiores ao encontrado em um Hospital Estadual Paulista onde 15,4\% dos óbitos se associaram à infecção hospitalar, sem restringir o setor de ocorrência ${ }^{20}$. Ao se comparar com dados gerais, nota-se que se encontram acima da média mundial $(23,0 \%)^{21}$ e não reflete a transição epidemiológica acelerada que foi divulgada em 2014 pelo Ministério da Saúde, em que as Causas Infecciosas não figuravam sequer entre as três primeiras causas de óbito ${ }^{16}$.

Outro dado a ser explorado é a progressão de Causas Infecciosas quando analisado o encadeamento dos eventos patológicos que culminaram com o óbito. Verificou-se que $11,3 \%$ ( $n=$ 550) dos pacientes apresentaram infecção como Causa Básica, $14,2 \%(n=273)$ como Causa Consequencial3, 25,5\% ( $n=42)$ como Causas Consequenciais 2, e 30,7\% ( $n=1476$ ) como Causa Imediata. Tal dado sugere que a infecção que entrou na cadeia de eventos responsáveis pela morte do paciente foi adquirida no Hospital. Para fins de comparação, no hospital paulista citado anteriormente, dos $15,4 \%$ dos óbitos por infecção, $8,7 \%$ se relacionaram diretamente com a infecção adquirida no hospital ${ }^{20}$. Outro estudo em um Hospital Universitário no Paraná mostrou uma incidência média mensal de 10,1\% de infecções hospitalares entre todos os pacientes internados ${ }^{22}$. Atualmente, a infecção hospitalar é uma das principais causas de mortalidade nosocomial, podendo estar associada a doenças graves, intervenções médicas e cirúrgicas e complicações a elas relacionadas ${ }^{20}$.

Ainda em relação às Causas Imediatas de óbito, o valor encontrado para as Causas Mal Definidas (17,6\%; $n=847)$, é superior ao preconizado pelo Ministério da Saúde (menor que $10 \%)^{23}$. Esse dado revela que, em $17,6 \%$ dos casos não se soube a causa de morte dentro do hospital, percentual que é superior ao encontrado em um Hospital Municipal de Belém do Pará $(15, \%)^{24}$, bem como às médias Brasileira e Nordestina em 2010 , em $8,5 \%$ e $8,1 \%$, respectivamente ${ }^{10}$. Quando somadas as duas principais Causas Imediatas de óbito: Causas Infecciosas $(30,7 \% ; n=1476)$ e Causas Mal Definidas $(17,6 \% ; n=847)$, notase que elas foram responsáveis por quase metade $(48,3 \% ; n=$ 2323) das mortes. É um dado que alerta para problemas como o desconhecimento das causas de morte dentro de um hospital que é referência estadual ${ }^{10,20}$.

Por meio da relação entre as Causas Básica e Imediata, foi possível avaliar a distribuição e a concentração de $77,4 \%(n=4220)$ dos óbitos constantes nas fichas da OPO, havendo, portanto, perdas no registro. Assim, viu-se que em $22,6 \%(n=1235)$ de casos registrados, as variáveis Causa Básica e Causa Imediata não foram preenchidas, reforçando a situação de desconhecimento do encadeamento de eventos patológicos que culminam com o óbito, uma vez que se trata da relação entre o motivo de entrada no hospital e o desfecho do atendimento. Essa análise ganha destaque ao mostrar o impacto das Causas Infecciosas na mortalidade do HUSE, pois foram a principal Causa Imediata em oito das onze categorias de Causa Básica, exceto naqueles pacientes que já apresentavam infecção como Causa Básica.

Os dados obtidos também levantam outra problemática: os pacientes acometidos por INF4 e DAR4 apresentaram INF1 como Causa Imediata em mais de $50 \%$ dos óbitos (INF4, 62,4\%; DAR4, 60,7\%). Em relação às doenças do aparelho respiratório, podemos destacar o rotineiro uso de procedimentos invasivos, como a ventilação mecânica, que favorece o desenvolvimento de Pneumonia Hospitalar ${ }^{25}$, o que também foi encontrado em outros dois estudos ${ }^{22,26}$. Porém, vale destacar que um estudo no qual $87,8 \%$ das pneumonias hospitalares estavam associadas à ventilação mecânica não encontrou correlação estatisticamente significativa entre pneumonia e ventilação mecânica ${ }^{20}$, de modo que os procedimentos invasivos isoladamente parecem não explicar o percentual aqui encontrado. Diante da relação entre as Causas de óbito que foram detectadas, destaca-se a importância das Causas infecciosas e das Causas Mal Definidas na mortalidade do HUSE, o que sugere que boa parte dos motivos de morte são desconhecidos nesse hospital.

O presente estudo apresentou limitações que devem ser consideradas para análise. Não se averiguaram informações importantes como o tempo de permanência no hospital e o internamento em UTI, o que poderia ajudar a entender algumas características da mortalidade intra-hospitalar. Além disso, não se compararam os dados coletados na OPO/HUSE com os das DOs do hospital, os quais auxiliariam na determinação de quão relevante é o acréscimo de informações que a utilização da OPO pode fornecer ao estudo da Mortalidade.

Por fim, sugere-se que sejam conduzidas novas pesquisas para o acompanhamento do perfil em setores específicos do Hospital como as áreas do Pronto-Socorro, UTI e setores pediátricos. Tais estudos voltados a contextos específicos contribuiriam com a elucidação do impacto das infecções em nível setorial.

\section{CONSIDERAÇÕES FINAIS}

As Doenças Infecciosas e as Causas Mal Definidas exibem notável impacto na mortalidade hospitalar do HUSE. Com isso, percebeu-se que os dados obtidos nesse estudo, além de mostrarem a viabilidade do uso de registros da OPO como parâmetro de avaliação da mortalidade hospitalar, podem também ser vistos como um problema a ser discutido, além de reiterarem a necessidade de melhora nos registros hospitalares e do Sistema de Informação.

\section{AGRADECIMENTOS}

Agradecimentos: Ao Ministério da Saúde pelo apoio financeiro por meio do Plano de Educação pelo Trabalho para Saúde/ Redes de Urgência e Emergência-PET-SAÚDE/RUE (Edital SGTES, no 11/2013). 


\section{REFERÊNCIAS}

1. World Health Organization. The top 10 causes of death. [citado 2015 Set 14]. Disponível em: http://www.who.int/mediacentre/factsheets/fs310/en/index4. html.

2. World Health Organization.World health statistics 2015. [ citado 2015 Set 14]. Disponível em: http://www.who.int/gho/publications/world_health_ statistics/2015/en/.

3. Oliveira PPV, Silva GA, Curado MP, Malta DC, Moura L. Cad.Saúde Pública 2014 Jan-Fev; 30(2):296-304. doi: http://dx.doi.org/10.1590/0102-311X00024813.

4. Koetsier A, Peek N, de Jonge E, Dongelmans DA, Van Berkel G, de Keizer NF. Reliability of In-hospital Mortality as a Quality Indicator in Clinical Quality Registries. A Case Study in an Intensive Care Quality Register. Methods Inf Med 2013; 52(5):432-440. doi: 10.3414/ME12-02-0070. PubMed PMID 23807704.

5. Feudtner C, Berry JG, Parry G, Hain P, Morse RB, Slonim AD, et al. Statistical Uncertainty of Mortality Rates and Rankings for Children's Hospitals. Pediatrics 2011 Oct; 128(4): 966-972.

6. Ministério da Saúde (Brasil). Secretaria de Atenção à Saúde. Departamento de Análise de Situação de Saúde. Manual de instruções para o preenchimento da declaração de óbito. Brasília: Ministério da Saúde; 2011. (Série A. Normas e Manuais Técnicos).

7. Organização Mundial da Saúde. Classificação estatística internacional de doenças e problemas relacionados a saúde - CID-10: 10ạ revisão. 8 ed. São Paulo: EDUSP; 2000. v.1, p.1184.

8. Lucena L, Cagliari GHB, Tanaka J, Bonamigo EL. Declaração de óbito: preenchimento pelo corpo clínico de um hospital universitário. Rev Bioét 2014; 22 (2):318-24. doi: http://dx.doi.org/10.1590/1983-80422014222013.

9. Cascão AM, Costa AJL, Kale PL. Qualidade da informação sobre mortalidade numa coorte de diabéticos - Estado do Rio de Janeiro, 2000 a 2003. Rev. bras. epidemiol 2012; 15(1):134-142. doi: http://dx.doi.org/10.1590/S1415$790 \times 2012000100012$.

10. Lima EEC, Queiroz BL. Evolution of the deaths registry system in Brazil: associations with changes in the mortality profile, under-registration of death counts, and ill-defined causes of death. Cad Saúde Pública 2014 Aug; 30(8):1721-1730. PubMed PMID 25210911.

11. Gomes AS, Kluck MM, Fachel JMG, Riboldi J. Fatores associados à mortalidade hospitalar na rede SUS do Rio Grande do Sul, em 2005: aplicação de modelo multinível. Rev. bras. epidemiol. 2010 Set;13(3):533-542. doi: http:// dx.doi.org/10.1590/S1415-790X2010000300016.

12. Vieira MS. Perfil geográfico e clínico de pacientes admitidos na UTI através da Central de Regulação de Internações Hospitalares. Comun ciênc saúde 2011; 22(3):201-210.

13. Obermeyer Z, Abujaber S, Makar M, Stoll S, Kayden SR, Wallis LA, et al. Emergency care in 59 low- and middle-income countries: a systematic review. Bull World Health Organ 2015; 93:577-586. doi: http://dx.doi.org/10.2471/ BLT.14.148338.
14. Zhou M, Wang H, Zhu J, Chen W, Wang L, Liu S, et al. Cause-specific mortality for 240 causes in China during 1990-2013: a systematic subnational analysis for the Global Burden of Disease Study 2013. Lancet. 2016 Jan 16; 387(10015): 25172. doi: 10.1016/S0140-6736(15)00551-6. PubMed PMID 26510778.

15. Cavalini LT, Cruz PS, Silva GM, Silva IF. Perfil da assistência em um Hospital Universitário: informações do registro hospitalar de câncer, 2000-2009. Rev bras. cancerol. 2012 Abr-Jun; 58(2):153-161.

16. Ministério da Saúde (Brasil). Secretaria de Vigilância em Saúde. Departamento de Análise de Situação em Saúde. Saúde Brasil 2013: uma análise da situação de saúde e das doenças transmissíveis relacionadas à pobreza. Brasília: Ministério da Saúde; 2014. p. 83-109.

17. Mendes JDV, Cecilio MAM. Tendências regionais da mortalidade por câncer no estado de São Paulo 2000 a 2010. BEPA 2012; 9(104):24-45.

18. Waiselfisz J J. Mapa da violência 2013: acidentes de trânsito e motocicletas. Rio de Janeiro: Centro Barsileiro de Estudos Latino- Americanos; 2013. p. 20-38.

19. Mascarenhas MDM, Barros MBA. Evolução das internações hospitalares por causas externas no sistema público de saúde - Brasil, 2002 a 2011. Epidemiol. Serv. Saúde 2015 Mar; 24(1):19-29. doi: http://dx.doi.org/10.5123/S167949742015000100003.

20. Guimarães AC, Donalisio MR, Santiago THR, Freire JB. Óbitos associados à infecção hospitalar, ocorridos em um hospital de Sumaré-SP, Brasil. Rev bras enferm. 2011 Set-Out; 64(5):864-9. doi: http://dx.doi.org/10.1590/S003471672011000500010 .

21. Adhikari NKJ, Fowler RA, Bhagwanjee S, Rubenfeld GD. Critical care and the global burden of critical illness in adults. Lancet. 2010 Oct; 376(9749):1339-46. doi: http://dx.doi.org/10.1016/S0140-6736(10)60446-1.

22. Izaias EM, Dellaroza MSG, Rossaneis MA, Belei RA. Custo e caracterização de infecção hospitalar em idosos. Ciênc saúde coletiva 2014 Ago;19(8):3395-3402. doi: http://dx.doi.org/10.1590/1413-81232014198.12732013.

23. Ministério da Saúde (Brasil). Secretaria de Atenção à Saúde. Caderno de diretrizes, objetivos, metas e indicadores 2013-2015. Brasília: Ministério da Saúde; 2013.

24. Veras,AP. Esclarecimento de óbitos por causas mal definidas, registradas no Hospital do Pronto Socorro Municipal Dr. Humberto Maradei Pereira, município de Belém do Pará, no ano de 2008[Dissertação]. Belém (Pará). ENSP Sergio Arouca; 2011.

25. Kalanuria AA, Zai W, Mirski M. Ventilator-associated pneumonia in the ICU. Crit Care 2014; 18(2): 208. doi: 10.1186/cc13775.

26. Juncal VR, Britto LA Neto, Camelier AA, Messeder OHC, Farias AMC. Impacto clínico do diagnóstico de sepse à admissão em UTI de um hospital privado em Salvador, Bahia. J bras pneumol. 2011 Jan-Fev; 37(1):85-92. doi: http://dx.doi. org/10.1590/S1806-37132011000100013.

\section{Como citar este artigo/How to cite this article:}

Alves ATLS, Faro A. Perfil epidemiológico da mortalidade em um hospital de urgência em Sergipe. J Health Biol Sci. 2016 Abr-Jun; 4(2):95-101. 